\title{
Omental Patching and Purse-String Endosuture Closure after Endoscopic Full-Thickness Resection in Patients with Gastric Gastrointestinal Stromal Tumors
}

\author{
Faisal Inayat', Aysha Aslam², Mathew D. Grunwald ${ }^{3}$, Qulsoom Hussain ${ }^{4}$, Abu Hurairah $^{3}$ and Shahzad Iqbal ${ }^{5}$ \\ ${ }^{1}$ Allama lqbal Medical College, Lahore, Pakistan, ${ }^{2}$ Harvard Medical School, Boston, MA, USA, ${ }^{3}$ SUNY Downstate Medical Center, Brooklyn, \\ NY, USA, ${ }^{4}$ Shifa College of Medicine, Islamabad, Pakistan, ${ }^{5}$ Hofstra-Northwell School of Medicine, Hempstead, NY, USA
}

Gastrointestinal stromal tumors (GISTs) are the most common mesenchymal tumors of the gastrointestinal tract, primarily arising from the stomach. With the widespread utilization of and technical advancements in endoscopy, gastric GISTs are being increasingly detected at an early stage, enabling complete endoscopic resection. Endoscopic full-thickness resection (EFTR) is an advanced technique that has been recognized as a treatment tool for neoplasms in the digestive tract in selected patients. Although a number of methods are available, closing large iatrogenic defects after EFTR can be a concern in clinical practice. If this potential problem is appropriately solved, patients with gastric GISTs would be suitable candidates for resection utilizing this technique. To our knowledge, this is the first study to propose omental patching and purse-string endosuture closure following EFTR as a feasible endoscopic option in patients with gastric GISTs. Clin Endosc 2019;52:283-287

Key Words: Omentum; Endoscopic full-thickness resection; Gastrointestinal stromal tumors; Therapeutic endoscopy; Endoscopic suturing

\section{INTRODUCTION}

Gastrointestinal stromal tumors (GISTs) commonly exhibit unpredictable clinical behavior and may pose a therapeutic challenge. The efficacy and safety of endoscopic techniques for the removal of GISTs are well established. ${ }^{1}$ Endoscopic full-thickness resection (EFTR) is an advanced and feasible endoscopic technique in this regard; however, its ability to close larger defects may be insufficient. ${ }^{2}$ Endoscopic methods such as metallic clips and endoloops are useful for small iat-

\footnotetext{
Received: July 3, 2018 Revised: August 16, 2018

Accepted: August 20, 2018

Correspondence: Faisal Inayat

Allama Iqbal Medical College, Allama Shabbir Ahmad Usmani Road, Faisal Town, Lahore 54550, Pakistan

Tel: +92-349-667-7229, Fax: +92-042-9923-1443, E-mail: faisalinayat@hotmail. com

ORCID: https://orcid.org/0000-0001-7576-7319
}

(cc) This is an Open Access article distributed under the terms of the Creative Commons Attribution Non-Commercial License (http://creativecommons.org/ licenses/by-nc/3.0) which permits unrestricted non-commercial use, distribution, and reproduction in any medium, provided the original work is properly cited. rogenic perforations, but they carry technical limitations for treating large-sized defects. Although commercialized closure devices for flexible endoscopy, including the overstitch system and over-the-scope-clip (OTSC) system, have shown promising outcomes for the closure of fistulas, iatrogenic defects, and anastomotic leakage after surgery, their widespread availability remains a problem. ${ }^{2}$ To increase the safety and improve the clinical outcomes of EFTR, the availability of reliable endoscopic suturing techniques and devices for defect closure is important. Herein, we employed omental patching and purse-string endosuture closure as an innovative technique for full-thickness defects following EFTR in 3 patients with gastric GISTs without incurring additional costs. The patients recovered well without the need for subsequent surgical interventions.

\section{CASE REPORTS}

\section{Case 1}

An 80-year-old woman was referred to our medical center 
for the evaluation of worsening gastroesophageal reflux disease and postprandial bloating for several months. She had a formidable medical history of osteoarthritis, asthma, cerebrovascular accident (on aspirin), chronic reflux disease, and low back pain. Esophagogastroduodenoscopy (EGD) revealed an approximately $2-\mathrm{cm}$-diameter round submucosal lesion in the gastric fundus along the greater curvature located $8 \mathrm{~cm}$ distal to the gastroesophageal junction (Fig. 1A). The lesion was successfully resected using EFTR, which left a full-thickness defect in the gastric wall (Fig. 1B). Initial endoscopic measures to close the defect failed. Therein, a decision was made to use omental patching. A mesenteric fat plug was pulled into the gastric lumen and it was clipped using the sequential application of hemoclips in a zipper-like fashion (Fig. 1C). Since the defect was considerably large, slight slippage of the omental patch with the hemoclips was observed. Therefore, we subsequently used endosutures in a purse-string fashion to completely close the gastric defect (Fig. 1D). The OverStitch ${ }^{\mathrm{TM}}$ Endoscopic Suturing System (Apollo Endosurgery, Austin, TX, USA) attached to the proximal and distal ends of a dual-channel gastroscope (GIF-2TH180; Olympus Medical Systems, Center Valley, PA, USA) provided the endoluminal suturing. Continuous sutures were first placed around the margins of the defect, followed by successful cinching. The resected specimen was sent for histopathological analysis (Fig. 1E). The pathologic examination findings were in line with the biopsy results confirming the diagnosis of GIST (mitotic rate, $<5 / 50$ high-power fields [HPF]) and signified a successful EFTR with tumor-free resected margins. The patient's post-procedure recovery was unremarkable and she was discharged on day 3 of admission.

\section{Case 2}

A 79-year-old man was referred to our medical center for the evaluation of an incidentally discovered submucosal gastric mass. He had no prior gastrointestinal problems, except gastroesophageal reflux disease. His past medical history was remarkable for bradycardia, hypertension, hyperlipidemia, chronic kidney disease, benign prostatic hyperplasia, and gout. He had a 20 pack-years history of smoking. At presentation, his physical examination was significant for mild temporal wasting. EGD (GIF-2TH180; Olympus Medical Systems) revealed a large approximately $2.5-\mathrm{cm}$ intramural submucosal lesion in the gastric cardia along the greater curvature (Fig. 2A). Endoscopic ultrasonography revealed an $18.5 \times 13 \mathrm{~mm}$ endophytic submucosal lesion originating from the fourth

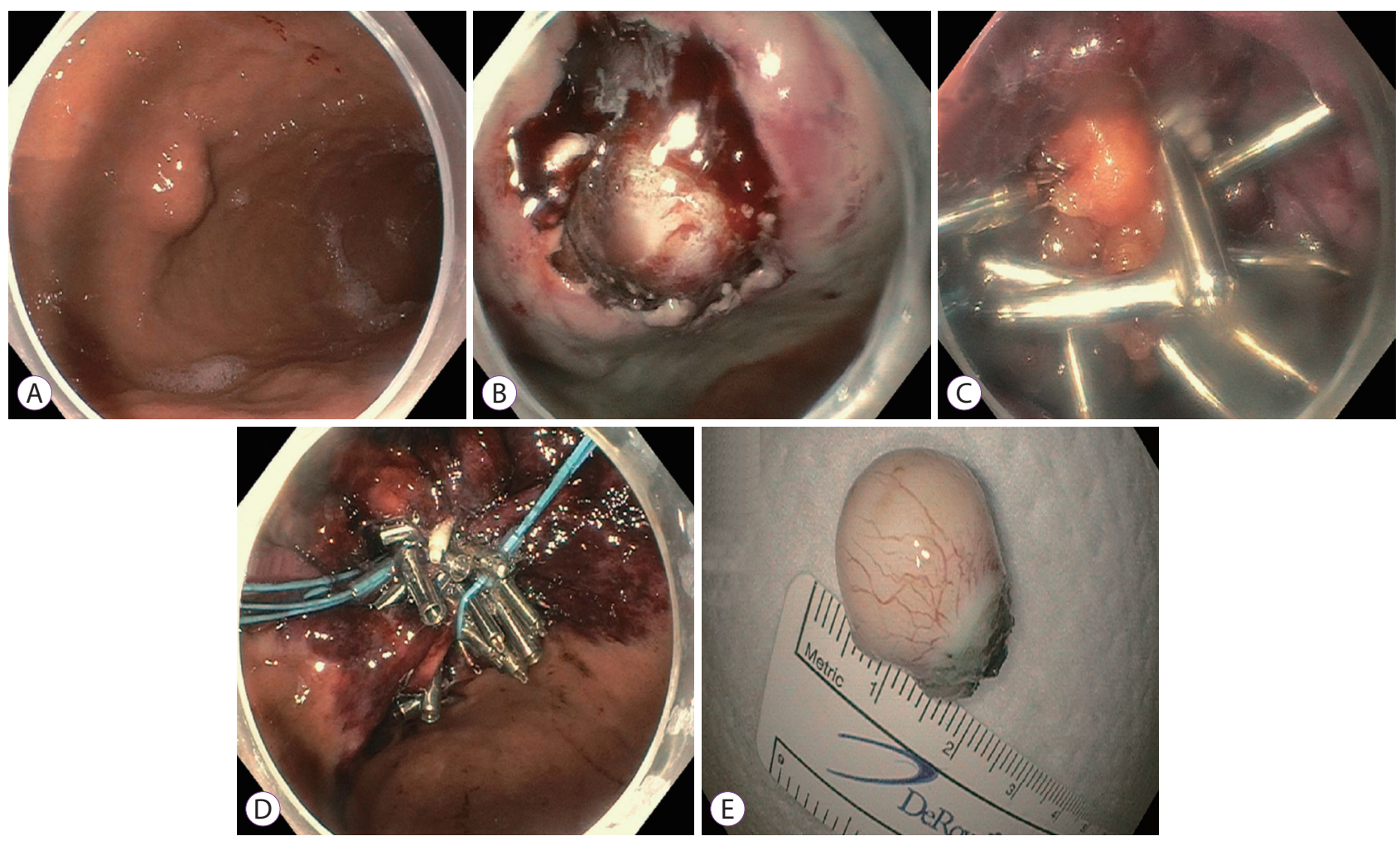

Fig. 1. (A) Esophagogastroduodenoscopy identified an approximately 2-cm round submucosal lesion located in the fundus/proximal body along the greater curvature of the stomach. (B) An iatrogenic full-thickness gastric wall defect was created by the endoscopic full-thickness resection. (C) The defect was successfully closed using omental fat patching. Several hemoclips were deployed to affix the fat plug to the gastric wall. (D) The hemoclips were then joined together by an endoloop. (E) Gross appearance of the resected specimen. A fleshy tan-white mass measuring $22 \mathrm{~mm}$ in diameter was resected and sent for pathological examination. 
echolayer (Fig. 2B). An uneventful EFTR was performed that created a large full-thickness wall defect. Initial attempts at closing the defect were unsuccessful due to slippage of sutures and a T-tag. Endoloop and hemoclips also failed. Thus, omental patching was planned. A mesenteric fat plug was pulled into the gastric lumen (Fig. 2C). Multiple hemoclips were deployed to closely appose the fat plug with the gastric wall (Fig. 2D). Due the large full-thickness defect, considerable risk of omental plug slippage was present with the use of hemoclips alone. Endosuturing in a purse-string fashion was then applied, which completely closed the defect (Fig. 2E). The resected specimen was sent for histopathological examination (Fig. 2F), confirming clear resection margins of the GIST lesion (mitotic rate, 6/50 HPF). The patient's recovery was uneventful and he was discharged from the hospital on day 4 of admission.

\section{Case 3}

A 54-year-old man was referred to our medical center for the evaluation of a submucosal gastric mass found on upper endoscopy performed for postprandial bloating. The Helicobacter pylori infection test was negative. Laboratory evalua- tion findings, including a complete blood profile and serum biochemistry were within normal limits. EGD (GIF-H190; Olympus Medical Systems) revealed a large approximately $3.2-\mathrm{cm}$ intramural submucosal lesion in the gastric cardia. The lesion was resected using EFTR, which resulted in a large full-thickness wall defect. Multiple attempts to close the defect utilizing various conventional and modern techniques failed. Therefore, omental patching was performed in which an omental fat plug was pulled into the gastric lumen and affixed to the gastric wall with hemoclips. However, adequate defect closure could not be achieved. Purse-string endosutures were then used around the clips to achieve reliable closure. The pathologic findings of the resected specimen were consistent with GIST (mitotic rate, $<5 / 50 \mathrm{HPF}$ ), and negative resection margins marked the clinical success of EFTR. The patient's recovery was uneventful and he was discharged from the hospital on day 4 of admission.

\section{DISCUSSION}

The mucosal wall defects after EFTR continue to be a major
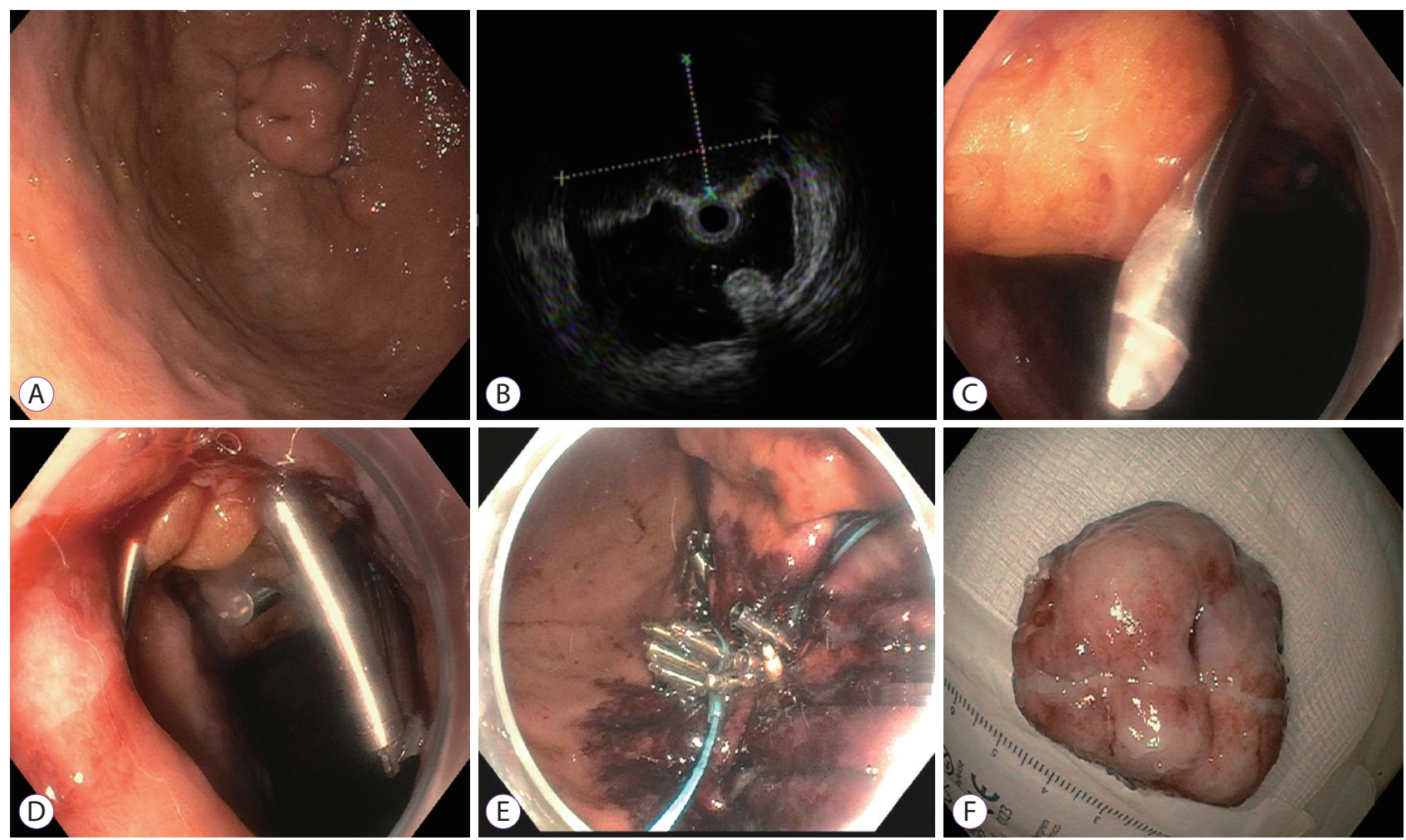

Fig. 2. (A) Esophagogastroduodenoscopy showing an approximately $2.5-\mathrm{cm}$ intramural submucosal lesion in the gastric cardia along the greater curvature. (B) Endoscopic ultrasound image showing an $18.5 \times 13 \mathrm{~mm}$ endophytic submucosal lesion originating from the muscularis propria. (C) The omental fat plug was pulled into the gastric lumen in an attempt to close the defect. (D) Multiple hemoclips were deployed to hold the fat plug within the gastric wall. (E) Endosutures were applied in a purse-string fashion around the hemoclips. (F) Gross morphology of the resected specimen. A multinodular mass with 28-mm ulcerations was resected and sent for pathological examination. 
concern for endoscopists. Backes et al. conducted a prospective study of 26 patients who underwent colorectal EFTR with a $100 \%$ technical success rate. ${ }^{3}$ However, $3(12 \%)$ patients developed complications within 48 hours, 1 of which was a perforation requiring laparoscopic suturing. ${ }^{3}$ Similarly, a systemic review of 10 animal studies showed a risk of post-EFTR perforations and defect-closure failure in $5 \%-55 \%$ of cases with an overall mortality rate of $4 \% .{ }^{4}$ Raju reported that the use of transabdominal sutures left a $3-4-\mathrm{cm}$ wall defect in 1 of 20 (5\%) animals, while an OTSC system for defect closure using twin graspers was employed in 9 of the 20 animals (45\%). von Renteln et al. reported defect-closure failure in 11 of 20 (55\%) animals, especially in cases of colostomies $>2.9 \mathrm{~cm}$ in diameter. ${ }^{6}$ Therefore, the closure of large defects is relatively difficult, which decreases the procedural success of EFTR.

In current practice, small gastrointestinal defects can be closed using multiple metallic clips; however, technical limitations have been noted for relatively larger defects. Ye et al. implicated the use of a combination of endoloops with endoclips for defect closure. ${ }^{7}$ Furthermore, the clip-withthread and loop-assisted methods were used by Lu et al. in 62 patients with submucosal tumors of the gastric fundus. ${ }^{8}$ These methods used traction devices that maintained the optimal endoscopic visibility resulting in faster and safer gastric EFTR in difficult anatomic locations, but intraoperative pneumoperitoneum occurred in both the thread-with-clip group and loop-assisted group (23\% and $18 \%$, respectively) that needed abdominal puncture to relieve free air. ${ }^{8}$ In a study by Guo and colleagues, OTSC system was employed in combination with metallic clips with promising outcomes. ${ }^{9}$ However, it is notable that delayed perforation has been reported even with the OTSC method. ${ }^{2}$ A consensus on the choice of an ideal defect-closure technique has yet to be reached upon, especially in patients with larger lesions.

To the best of our knowledge, the present study represents the first report of omental patching using a purse-string enendosuture method in an attempt to close EFTR-related defects for small-to-average GIST lesions. Omental patching was previously used to repair peptic ulcer perforations in pyloric, pre-pyloric, and duodenal regions with successful outcomes. ${ }^{1011}$ However, the published medical literature is sparse on its endoscopic use for EFTR-related defects in patients with GIST. The technique was discussed previously for complete endoscopic closure of gastric perforations induced by the endoscopic resection of early gastric cancers but not many cases or studies have been performed to validate it. ${ }^{12}$

In this study, all lesions were $>2 \mathrm{~cm}$ and EFTR-related iatrogenic defects were closed endoscopically utilizing this new method. Although we used omental patching with hemoclipping alone first, the defects were not adequately closed. Thus, endosuturing was used. The technique of combined omental

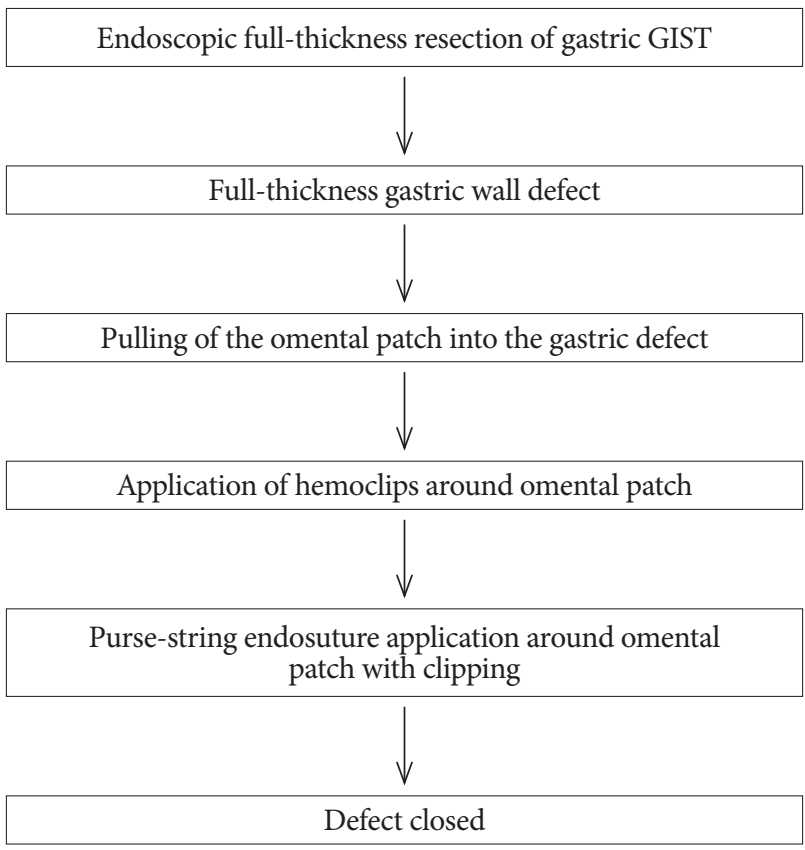

Fig. 3. Flowchart showing the omental patching and purse-string technique for closing endoscopic full-thickness resection-related gastric defects in patients with gastrointestinal stromal tumors (GISTs).

Table 1. Data of the Patients Recruited into This Study

\begin{tabular}{lccccccccc}
\hline Case & Age & $\begin{array}{c}\text { Gen- } \\
\text { der }\end{array}$ & $\begin{array}{c}\text { Lesion } \\
\text { site }\end{array}$ & $\begin{array}{c}\text { EFTR in- } \\
\text { dication }\end{array}$ & $\begin{array}{c}\text { Resected speci- } \\
\text { men size }(\mathbf{m m})\end{array}$ & $\begin{array}{c}\text { Failed closure } \\
\text { attempts before } \\
\text { omental patching }\end{array}$ & $\begin{array}{c}\text { Immunohistochemical } \\
\text { features }\end{array}$ & $\begin{array}{c}\text { Hospital } \\
\text { stay } \\
\text { (days) }\end{array}$ & $\begin{array}{c}\text { Compli- } \\
\text { cations }\end{array}$ \\
\hline 1 & 80 & F & $\begin{array}{c}\text { Gastric } \\
\text { fundus }\end{array}$ & GIST & 22 & 2 & $\begin{array}{c}\text { CD117 and DOG1 positive; actin } \\
\text { negative }\end{array}$ & None \\
2 & 79 & M & $\begin{array}{c}\text { Gastric } \\
\text { cardia }\end{array}$ & GIST & 28 & 3 & $\begin{array}{c}\text { CD117 and DOG1 positive; ac- } \\
\text { tin, S100, and desmin negative }\end{array}$ & 4 & None \\
3 & 54 & M & $\begin{array}{c}\text { Gastric } \\
\text { cardia }\end{array}$ & GIST & 35 & 3 & $\begin{array}{c}\text { CD117 and DOG-1 diffusely pos- } \\
\text { itive; SMA patchy and weakly } \\
\text { positive; desmin negative }\end{array}$ & 4 \\
\hline
\end{tabular}

EFTR, endoscopic full-thickness resection; GIST, gastrointestinal stromal tumor. 
patching and purse-string endosuturing is illustrated in Fig. 3. The lesions in Cases 1 and 3 were low-grade GISTs, while that in Case 2 was moderate-grade. The participants' data are summarized in Table 1 . None of the cases were associated with peri- or postoperative complications. After multidisciplinary consultations, no further surgical or oncological therapies were needed. At the 3-month follow-up, contrast-enhanced computed tomography (CT) abdomen and/or EGD showed interval resections of gastric lesions with multiple hemoclips in the resection bed. The patients were followed clinically in an outpatient setting with subsequent surveillance abdominal CT or EGD according to National Comprehensive Cancer Network guidelines. No GIST recurrence was noted. The follow-up range was 8-29 months. There were no signs of perforation or other complications, marking $100 \%$ procedural and durable clinical success of the omental patching and pursestring endosuture closure method. Hence, this technique may result in a low cost, shortened hospital stay, decreased need for human resources, and lower risk of complications when performed by expert endoscopists. Large multicenter studies are warranted to stratify the risk of perforation and other post-procedural complications following the use of omental patching with the purse-string endoscopic closure technique, especially in patients with large and difficult-to-access defects created by EFTR.

\section{Conflicts of Interest}

The authors have no financial conflicts of interest.

\section{Acknowledgement}

This case series was presented as an abstract at Digestive Disease Week, May 6-9, 2017, in Chicago, IL, USA.

\section{REFERENCES}

1. Bai J, Wang Y, Guo H, Zhang P, Ling X, Zhao X. Endoscopic resection of small gastrointestinal stromal tumors. Dig Dis Sci 2010;55:1950-1954.

2. Mori $\mathrm{H}$, Kobara $\mathrm{H}$, Nishiyama N, Masaki T. Current status and future perspectives of endoscopic full-thickness resection. Dig Endosc 2018;30 Suppl 1:25-31.

3. Backes Y, Kappelle WFW, Berk L, et al. Colorectal endoscopic full-thickness resection using a novel, flat-base over-the-scope clip: a prospective study. Endoscopy 2017;49:1092-1097.

4. Brigic A, Symons NR, Faiz O, Fraser C, Clark SK, Kennedy RH. A systematic review regarding the feasibility and safety of endoscopic full thickness resection (EFTR) for colonic lesions. Surg Endosc 2013;27:3520-3529.

5. Raju GS. Endoscopic clip closure of gastrointestinal perforations, fistulae, and leaks. Dig Endosc 2014;26 Suppl 1:95-104.

6. von Renteln D, Schmidt A, Vassiliou MC, Rudolph HU, Caca K. Endoscopic full-thickness resection and defect closure in the colon. Gastrointest Endosc 2010;71:1267-1273.

7. Ye LP, Yu Z, Mao XL, Zhu LH, Zhou XB. Endoscopic full-thickness resection with defect closure using clips and an endoloop for gastric subepithelial tumors arising from the muscularis propria. Surg Endosc 2014;28:1978-1983.

8. Lu J, Jiao T, Li Y, Zheng M, Lu X. Facilitating retroflexed endoscopic full-thickness resection through loop-mediated or rope-mediated countertraction (with videos). Gastrointest Endosc 2016;83:223-228.

9. Guo J, Liu Z, Sun S, et al. Endoscopic full-thickness resection with defect closure using an over-the-scope clip for gastric subepithelial tumors originating from the muscularis propria. Surg Endosc 2015;29:33563362.

10. Smith D, Roeser M, Naranjo J, Carr JA. The natural history of perforated foregut ulcers after repair by omental patching or primary closure. Eur J Trauma Emerg Surg 2018;44:273-277.

11. Karanjia ND, Shanahan DJ, Knight MJ. Omental patching of a large perforated duodenal ulcer: a new method. Br J Surg 1993;80:65.

12. Minami S, Gotoda T, Ono H, Oda I, Hamanaka H. Complete endoscopic closure of gastric perforation induced by endoscopic resection of early gastric cancer using endoclips can prevent surgery (with video). Gastrointest Endosc 2006;63:596-601. 